\title{
28 Research Soure \\ Innate lymphoid cells at the maternal-fetal interface: Implications in preterm labor
}

João Fernando Pereira Mendes ( $\boldsymbol{\nabla}$ jmendes@uc.pt)

Universidade de Coimbra https://orcid.org/0000-0002-4697-0542

Paulo Rodrigues Santos

Universidade de Coimbra

Ana Areia

Universidade de Coimbra

Jani Sofia Almeida

Universidade de Coimbra https://orcid.org/0000-0003-2024-0434

Vera Alves

Universidade de Coimbra

Manuel Santos-Rosa

Universidade de Coimbra

Anabela Mota-Pinto

Universidade de Coimbra https://orcid.org/0000-0002-0820-9568

\section{Research article}

Keywords: Preterm Labor, Inflammation, Innate Immune Response, Innate Lymphoid Cells, Preterm birth

Posted Date: July 18th, 2019

DOI: https://doi.org/10.21203/rs.2.11586/v1

License: (c) (1) This work is licensed under a Creative Commons Attribution 4.0 International License.

Read Full License 


\section{Abstract}

Background: Preterm labor (PTL) is one of the major causes of neonatal morbidity and mortality worldwide. It is commonly accepted that the act of giving birth is the final step in a proinflammatory signaling cascade, orchestrated by an intrauterine milieu coupled to hormonal cues. Consequently, the inflammatory process plays a pivotal role during the pathogenesis of human labor, both in term and preterm deliveries. The aim of this work was to analyze the abilities of innate lymphoid cells (ILCs), specifically ILC3 NCR- cells, to act as proinflammatory mediators and to elucidate their role in pregnancy. Accordingly, we hypothesize that altered ILC numbers may prompt preterm labor (PTL). Methods: We analyzed thirteen full term labor (FTL) and seven PTL pregnant women for the presence of ILCs. ILCs were isolated and characterized from maternal peripheral blood, maternal-fetal interface, and cord blood samples, using flow cytometry. For group analysis, two-way ANOVA was performed. Each data set was analyzed using student t-test with a confidence interval of 95\%. Results: A tendency for decreased ILCs numbers in PTL samples compared to those in FTL samples was observed. Additionally, ILC3 NCR- cells were significantly increased in both PTL and FTL groups, when compared to ILC3NCR+. Conclusion: Our results support a potential inflammatory role of ILC3 NCR- in the instigation of labor. Moreover our work highlights the importance of ILCs actions in the regulation of labor.

\section{Background}

In the past 5 years, an increasing number of studies have focused on a relatively new set of cells, termed innate lymphoid cells (ILCs). Commonly characterized as belonging to the innate component of the immune system, these cells are potentially of substantial interest for neonatal care, mainly for women in preterm labor (PTL).

PTL is one of the major causes of neonatal morbidity and mortality worldwide. In Portugal, of the 85,500 live-born children in $2015,8 \%$ were born premature(1). These numbers justify the growing scientific interest in this research, as the world eagerly awaits a new standard of care that might be implemented to allow reduce neonatal mortality due to PTL.

The gestational period is comprised of decidualization, placentation and fetal development and requires uterine quiescence and the production of both anti- and proinflammatory cytokines from maternal and fetal cells(2).

Indeed, throughout pregnancy, three distinct biological periods characterized by different immunological microenvironments occur; the first trimester comprises an inflammatory period, while the second trimester comprises an anti-inflammatory period (during which the fetus grows), and the third trimester also comprises an inflammatory period that leads to delivery(3).

Supported by previous work from our group(4)'(5), ILC populations appear to be an interesting target to further investigate, and reports of ILCs having diverse functions that range from inflammation, protection against infections and microorganisms, tumor surveillance and mediation of graft-versus-host disease 
have been published(6). As ILCs are thought to play a role in the innate immune response(7), the notion that they may be the origin of a composed inflammatory cascade has drawn our attention. In fact, ILCs can be divided into three distinct groups: ILC1, ILC2 and ILC3. Group 1 ILCs express the transcription factor T-bet and produce interferon- $\gamma(8)$. Group 2 ILCs highly express the transcription factor GATA-3 and produce interleukin 5 (IL-5), IL-9 and IL-13, important for helminth infections and allergies(9)'(10). Group 3 ILCs secrete IL-17 and IL-22 and express the transcription factor retinoic acid receptor-related orphan nuclear receptor gamma (RORYt) (11)'(12).

According to the literature, ILC3 cells may be of substantial interest for women in PTL since they not only express the natural cytotoxic receptor (NCR, which is thought to have a stimulatory effect on natural killer (NK) cell action) but also produce IL-22 and IL-17 under the control of the transcription factor RORyt (13), (14) and aryl hydrocarbon receptor (AHR)(15). In this context, IL-17 is substantially relevant since it promotes a proinflammatory state, which might trigger premature birth.

Additionally, IL-22 initiates cell cycle progression via the Jak/Stat pathway, thus contributing to tissue regeneration and homeostasis(16), which is of significant importance for placental maintenance.

Studies describing ILC populations during pregnancy or their role in PTL are still scarce. Vacca et al. published an extensive study that clearly identifies the ILC1 and ILC3 populations in the first trimester decidua. They demonstrated that these cells have the ability to produce proinflammatory cytokines, including IL-8, IL-22, IL-17A, TNF, and IFNY(17), and they concluded that ILC3 and ILC1 may play key roles in the implantation process together with tissue remodeling functions due to their secretion of proinflammatory and angiogenic factors. Conversely, $X u$ et al. showed that ILC2 is the most abundant population in the decidua, capable of producing Th2-type cytokines, such as IL- 4, IL- 5, and IL- 13. In that study, the authors suggest that the proinflammatory proprieties of ILC2 might underlie the pathological process prompting PTL(18). Specifically, $X u$ et al. argue that ILC populations dynamically change throughout pregnancy. In fact, they also detected ILC3 in the decidua parietalis, capable of producing IL17 and IL-22, suggesting that these cells may be responsible for inflammation-driven PTL. However, Xu et al. also propose that ILC3 can regulate inflammatory actions via the production of IL13.

The discrepancies in the previously published studies highlight the fact that further studies are needed to clarify the role of ILCs and the innate immune system in the pregnancy process and in abnormal situations, such as PTL. Thus, this study aimed to isolate and characterize ILC populations from the decidua basalis of pregnant women and compare the results with those in preterm birth samples. Based on the changes in the different ILC population numbers, we postulate that ILC3 might play a preponderant role in the instigation of labor.

\section{Methods}

Population. 
Female patients who planned to deliver at the Obstetric Department of Coimbra Hospital and University Centre (CHUC) were invited to participate in the study.

The study inclusion criteria consisted of pregnant women monitored by normal prenatal appointments and women presenting to the emergency room in labor. Inclusion criteria for control group comprised: healthy pregnant women attending normal prenatal appointments; full term singleton pregnancies, delivered after labor; and first prenatal appointment before $14^{\text {th }}$ week gestation. The inclusion criteria for the study group (PTL group) were as follows: admission to the Fetal Maternal Medicine Obstetric Department of CHUC with confirmed spontaneous preterm labor, singleton pregnancy, gestational age between 24 weeks +0 days and 36 weeks +6 days, intact amniotic membrane, cervical length $\leq 25 \mathrm{~mm}$ and the use of atosiban (competitive oxytocin receptor antagonist) for tocolysis (for contraction cessation).

Exclusion criteria were the following: multiple gestations, preterm rupture of membranes, chorioamnionitis, placenta previa or placental abruption, intrauterine growth restriction (IUGR), and preexistente diseases, namely: hypertension, diabetes, autoimmune diseases and allergies. Clinical chorioamnionitis was diagnosed based on clinical and laboratorial parameters like fever, maternal tachycardia, fetal tachycardia, maternal leukocytosis, uterine tenderness, foul-smelling amniotic fluid (AF); elevated maternal C-reactive protein and/or amniotic interleukin (IL)-6.

Women subjected to elective pre-labor Caesarean were not included as they have other medical etiologies, not focused in this work. The aim of this work was to describe ILC populations in the time of labor, and pave the way for further studies in the immunological events that trigger preterm labor.

Isolation of innate lymphoid cells

After delivery, the placenta was collected and rinsed in phosphate buffered saline (PBS, $\mathrm{Ca}^{2+}$-and $\mathrm{Mg}^{2+}$ free) (Corning ${ }^{\circledR}, N e w$ York, USA), to wash cloths and superfluous blood. Decidual tissue was dissected while soaking in $1 \times \mathrm{PBS}\left(\mathrm{Ca}^{2+}\right.$-and $\mathrm{Mg}^{2+}$-free). Excess villi and blood vessels were removed using finepoint scissors and forceps, washed with $1 \times \mathrm{PBS}\left(\mathrm{Ca}^{2+}\right.$-and $\mathrm{Mg}^{2+}$-free) and centrifuged at $400 \times \mathrm{g}$ for 5 min at room temperature. The supernatant was aspirated, and the tissue pellet was collected for dissociation. After dissociation, the tissue pellet was incubated for $45 \mathrm{~min}$ at $37^{\circ} \mathrm{C}$ in a $0.25 \mathrm{mg} / \mathrm{L}$ collagenase solution (Sigma-Aldrich ${ }^{\circledR}$, St. Louis, USA). After incubation, the cell suspension was passed through a $100 \mu \mathrm{m}$ cell strainer into a $50 \mathrm{ml}$ plastic tube. The $50 \mathrm{~mL}$ tube was filled with $1 \times \mathrm{PBS}\left(\mathrm{Ca}^{2+}\right.$-and $\mathrm{Mg}^{2+}$-free) and centrifuged at $400 \times \mathrm{g}$ for $5 \mathrm{~min}$ at room temperature. Next, $5 \mathrm{ml}$ of $20 \%$ Lymphoprep $^{\mathrm{TM}}$ density gradient media $(1.077+0.001 \mathrm{~g} / \mathrm{ml})$ (StemCell Technologies $\left.{ }^{\mathrm{TM}}\right)$, was placed into a $15 \mathrm{ml}$ plastic tube, and the cell suspension was slowly overlaid on top of the density gradient media. The tubes were filled with $1 \times$ PBS $\left(\mathrm{Ca}^{2+}\right.$-and $\mathrm{Mg}^{2+}$-free) and slowly centrifuged at $750 \times \mathrm{g}$ for $30 \mathrm{~min}$ at $4^{\circ} \mathrm{C}$ without the brake. Leukocytes were isolated from the interface between the density gradient media and the PBS

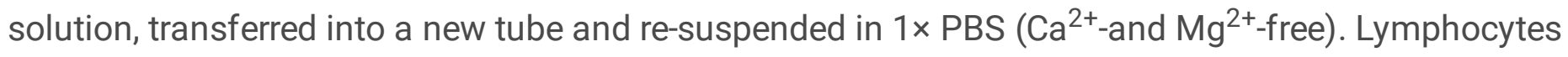
were isolated from the decidua basalis and adjacent tissue (villi) as described by Yi Xu et al.(19). Cells 
were counted on an Beckman coulter AcT Diff automatic cell counter (Beckman coulter, Brea, California, EUA), and a $100 \mu \mathrm{L}$ cell suspension containing $1 \times 10^{6}$ isolated lymphocytes was placed in a cytometry tube and labelled with primary antibodies (BD Biosciences, San Jose, USA). For lymphocyte discrimination, $\mathrm{CD}_{4} 5^{+}$and $\mathrm{CD}^{-}{ }^{-}$were used. Lineage-negative ( $\left.\mathrm{Lin}^{-}\right)$cells where labeled with $\mathrm{CD} 1, \mathrm{CD} 11 \mathrm{c}$,

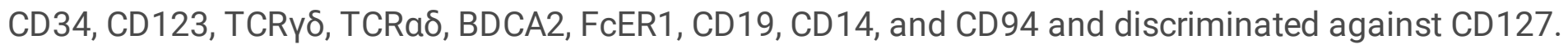
Cells expressing CD161 were then selected. To ascertain the different ILC populations, CD117, CRTH2 and NKp44 antibodies were used as described by Hazenberg et al.(20).

Stained samples were acquired on a FACS Canto II instrument (BD Biosciences, San Jose, USA) equipped with 3 lasers to allow multicolor detection with different fluorophores, using FACS DIVA software (BD Biosciences, San Jose, USA).

Statistical analysis

For group analysis, two-way ANOVA was performed using GraphPad® V7 software. Each data set was analyzed using student t-test analysis with a confidence interval of $95 \%$.

\section{Results}

In the current investigation, we analyzed thirteen full-term delivery samples and seven preterm delivery samples. In the full-term group, the median maternal age was 33 years $(26.7<95 \% \mathrm{Cl} 37.6)$, the median gestational age at delivery was 39 weeks $(38.1<95 \% \mathrm{Cl}$ 39.9) and the median birth weight was 3310 grams $(2855<95 \% \mathrm{Cl}<3682)$. In the preterm labor group, the median maternal age was 32 years $(20.0<$ $95 \% \mathrm{Cl}<38.0)$, the median gestational age at delivery was 35 weeks $(34.0<95 \% \mathrm{Cl} 36.0)$, and the median birth weight was 2690 grams $(2300<95 \% \mathrm{Cl} 3035)$.

As previously described, for the identification of ILCs, after isolating the lymphocyte population (Figure $1 \mathrm{~A}$ ), we utilized $\mathrm{CD} 3^{-} \mathrm{CD} 45^{+}$as the gating strategy(Figure 1B), and then selected $\mathrm{Lin}^{-} \mathrm{CD} 127^{+}$cells (Figure 1C). We next selected CD $161^{+}$cells (Figure 1D), and $\mathrm{CD} 117^{+}{ }^{+} \mathrm{CRTH} 2^{-}$cells were gated (Figure 1E). Finally, for the identification of ILC3 NCR ${ }^{+}$cells, we plotted CD117 $7^{+}$against NKp44 (Figure 1 F).

A decreasing tendency in the overall number of ILC populations was observed in the PTL group compared to that in the FTL samples (Figure 2A), and a tendency for a decrease in the ILC2 population, which was clearly decreased in PTL samples (Figure 2B).

When observing the ILC3 subsets, no significant differences between the ILC3 NCR ${ }^{-}$and ILC3 NCR ${ }^{+}$ populations were observed when comparing the PTL and FTL groups (Figure 2C). However, when comparing ILC3 NCR and ILC3 NCR ${ }^{+}$cells within the FTL group, the ILC3 NCR population was clearly increased ( $p<0.001$, student t-test with $95 \%$ confidence). The same comparison was also made between ILC3 NCR and ILC3 NCR ${ }^{+}$cells in the PTL group, and an increased ILC3 NCR population was also observed ( $p<0.05$, student t-test with $95 \%$ confidence). 


\section{Discussion}

These preliminary results regarding ILC3 populations demonstrate that ILCs are unequivocally present in the placentas of both FTL and PTL pregnant women, as we clearly observed these cells at the maternalfetal interface.

ILCs, specifically the ILC1 and ILC3 populations, have been described to play a preponderant role in tissue remodeling and homeostasis(21)'(22)'(7), which could partially explain the reduced numbers of ILCs observed in women in labor. Indeed, labor is the surrogate endpoint of pregnancy; thus, that support function is no longer needed.

When analyzing ILC populations in the FTL group (Figure 2B), the ILC2 and ILC3 populations tended to be increased in relation to the overall lymphocyte population, unlike the ILC1 population. Despite that ILC2 produces type 2 cytokines (IL-4, IL-5, IL-13) under the control of IL-25 and IL-33, important in helminth and extracellular parasitic infections(9), they can also elicit a strong inflammatory reaction(10). One aspect of IL-13's action is its ability to stimulate PGE2 in dendritic cells and macrophages(23), key mediators of labor initiation. Additionally, as mentioned by Spits et al. ILC3 NCR ${ }^{+}$cells produce mainly IL-22 and ILC3, while NCR cells produce IL-17(6), which is intriguing since IL-22 is considered a homeostatic cytokine that contributes to tissue and organ integrity. On the other hand, IL-17 mostly functions as an proinflammatory cytokine (24). However, $X u$ et al. reported that ILC3 cells also have the ability to produce IL-13, a finding that could also account for the instigation of labor if further confirmed. Herein, we found the ILC3 NCR population to be significantly elevated (compared to that of ILC3 NCR ${ }^{+}$), which is in accordance with the findings highlighted by Vacca et al. As that previous study showed, the ILC3 population can produce proinflammatory cytokines in decidua isolated from women in their first trimester of pregnancy(17), highlighting a potentially important role for ILC3 cells in the first stages of pregnancy.

Nonetheless, we cannot reject the assumption that Th2type responses were orchestrated by ILC2 since these cells are capable of presenting antigens to $\mathrm{T} \mathrm{CD} 4^{+}$cells and induce proliferation towards a Th2 phenotype in an IL-2-dependent manner(9). This is a remarkable finding since it proposes crosstalk between the innate and adaptive immune systems(25)'(10)'(26). However, ILC3 has been shown to express MHC II molecules, promoting a T cell-mediated response(27). This fact may elucidate the role of ILCs in mounting an adaptive immune response, potentially representing a tolerance mechanism towards the fetus, which is of substantial interest. Our study does present some limitations that must be considered, because our study utilized only a small sample number, our results should be interpreted with caution. To address this issue, future studies should include a larger number of women, and we will next direct our attention to a comprehensive analysis of cytokine release throughout pregnancy.

Conclusion.

A strong point of this study is its corroboration of the existing hypothesis that an inadequate inflammationtriggered immunological response prompts PTL, suggesting a key role of ILCs in this 
process; unfortunately, numerous mechanisms underlying ILC actions in pregnancy remain to be ascertained.

As mentioned previously, implantation and parturition are considered proinflammatory processes(2). Considering the different inflammatory periods of pregnancy, considering that ILC populations may fluctuate and differ throughout pregnancy is reasonable. In this sense, studying an abnormal inflammatory response in PTL becomes imperative. Human studies in this field are scarce, and the prevalence of PTL is increasing, leading to substantial increments in children handicaps and hospital costs.

Our results are encouraging, as they suggest a role of ILCs in the regulation of labor. Indeed, the data presented herein suggest that labor might be characterized by decreased tissue remodeling and repair functions, accompanied by a marked inflammatory response. Thus, we aim to expand the current knowledge on the immunology of pregnancy by focusing on ILC fluctuations during the pregnancy process and carefully analyzing cytokine release profiles.

\section{Abbreviations}

PTL - Preterm labor

FTL - Full-term labor

ILC - Innate lymphoid cells

IL - Interleukin

NCR-Natural cytotoxic receptor

\section{Declarations}

\section{Ethical approval.}

A written protocol encompassing the research details and general objectives of the proposal was approved by the hospital ethics committee (reference N.0179/CES from 19-10-2016), and informed consent was obtained from each patient under strict ethical protocols.

\section{Availability of data and materials}

The datasets used and/or analyzed during the current study are available from the corresponding author on reasonable request.

\section{Conflict of Interest}


The authors declare that they have no conflict of interest.

\section{Funding}

This work was supported by Centro de Investigação em Meio Ambiente, Genética e Oncobiologia (CIMAGO) of the Faculty of Medicine, University of Coimbra, (Coimbra, Portugal) [Grant Number 06/14]

\section{Author contribution:}

Mendes: Data collection, scientific analysis manuscript writing and editing.

Rodrigues Santos: Writing and supervising all the scientific analysis.

Areia: Writing, performing the medical sample collection and supervising patient clinical data.

Almeida: Data analysis.

Alves: Supervised the experiment design and technical support.

Santos-Rosa: Writing and supervision of scientific content.

Mota-Pinto: Coordination of research group, supervision of scientific content and project development.

\section{Acknowledgments}

The authors would like to thank the Centro de Investigação em Meio Ambiente, Genética e Oncobiologia (CIMAGO) of the Faculty of Medicine of Coimbra University for funding this study.

\section{References}

1. Demographic statistics- Birth and Mortality indicators. Statistics Portugal - Instituto Nacional de Estatística (INE) [Internet]. Available from: www.ine.pt

2. Sharma S, Thaxton JE, Nevers TA. TLR-mediated preterm birth in response to pathogenic agents. Infect Dis Obstet Gynecol. 2010;2010.

3. Hanssens S, Salzet M, Vinatier D. Immunological aspect of pregnancy. J Gynecol Obs Biol Reprod. 2012;41(7):595-611. 
4. Areia AL, Vale-pereira S, Vaz-ambrósio A, Alves V, Rodrigues-santos P, Rosa MS, et al. Does progesterone administration in preterm labor influence Treg cells? J Perinat Med. 2015;

5. Areia A, Vale-Pereira S, Alves V, Rodrigues-Santos P, Santos-Rosa M, Moura P, et al. Can membrane progesterone receptor on T regulatory cells explain the ensuing human labour? J Reprod Immunol. 2016;113:22-6.

6. Spits H. Human innate lymphoid cells. J Allergy Clin Immunol. 2016;138(5):1265-76.

7. Artis D, Spits H. The biology of innate lymphoid cells. Nature. 2015;517(7534):293-301.

8. Bernink JH, Peters CP, Munneke M, Te Velde AA, Meijer SL, Weijer K, et al. Human type 1 innate lymphoid cells accumulate in inflamed mucosal tissues. Nat Immunol. 2013;14(3):221-9.

9. Oliphant CJ, Hwang YY, Walker JA, Salimi M, Wong SH, Brewer JM, et al. MHCIl-mediated dialog between group 2 innate lymphoid cells and CD4+ T cells potentiates type 2 immunity and promotes parasitic helminth expulsion. Immunity [Internet]. 2014;41(2):283-95. Available from:

http://dx.doi.org/10.1016/j.immuni.2014.06.016

10. Halim TYF, Steer CA, Mathä L, Gold MJ, Martinez-Gonzalez I, McNagny KM, et al. Group 2 innate lymphoid cells are critical for the initiation of adaptive $T$ helper 2 cell-mediated allergic lung inflammation. Immunity. 2014;40(3):425-35.

11. Bando JK, Colonna M. Innate lymphoid cell function in the context of adaptive immunity. Nat Immunol. 2016;17(7):783.

12. Serafini N, Klein Wolterink RGJ, Satoh-Takayama N, Xu W, Vosshenrich CAJ, Hendriks RW, et al. Gata3 drives development of RORyt ${ }^{+}$group 3 innate lymphoid cells. J Exp Med [Internet]. 2014;211(2):199-208. Available from: http://www.jem.org/lookup/doi/10.1084/jem.20131038

13. Pavert SA Van De, Vivier E, Marseille-luminy CI De, Um UA, Inserm U, Umr C. Differentiation and function of group 3 innate lymphoid cells, from embryo to adult. 2015;28(1):35-42.

14. Spits H, Cupedo T. Innate Lymphoid Cells: Emerging Insights in Development, Lineage Relationships, and Function. Annu Rev Immunol [Internet]. 2012;30(1):647-75. Available from: http://www.annualreviews.org/doi/10.1146/annurev-immunol-020711-075053

15. Qiu J, Heller JJ, Guo X, Chen Z-E, Fish K, Fu Y-X, et al. Article The Aryl Hydrocarbon Receptor Regulates Gut Immunity through Modulation of Innate Lymphoid Cells. Immunity. 2011;36(1):92-104.

16. Dickinson AM. Interleukin-22 in Graft-Versus-Host Disease after Allogeneic Stem Cell Transplantation. 2016;7(April):1-9. 
17. Vacca P, Montaldo E, Croxatto D, Loiacono F, Canegallo F, Venturini PL, et al. Identification of diverse innate lymphoid cells in human decidua. Mucosal Immunol [Internet]. 2015;8(2):254-64. Available from: http://dx.doi.org/10.1038/mi.2014.63

18. Xu Y, Romero R, Miller D, Silva P, Panaitescu B, Theis KR, et al. Innate lymphoid cells at the human maternal-fetal interface in spontaneous preterm labor. Am J Reprod Immunol [Internet]. 2018; (January):e12820. Available from:

http://www.ncbi.nlm.nih.gov/pubmed/29457302\%0Ahttp://doi.wiley.com/10.1111/aji.12820

19. Yi Xu, Olesya Plazyo, Roberto Romero, Sonia S. Hassan and NG-L. Isolation of leukocytes from the human maternal-fetal interface. J Vis Exp. 2015;27(6):316-24.

20. Hazenberg MD, Spits H. Review Article Human innate lymphoid cells. Blood. 2014;124(5):700-10.

21. Hanna J, Goldman-Wohl D, Hamani Y, Avraham I, Greenfield C, Natanson-Yaron S, et al. Decidual NK cells regulate key developmental processes at the human fetal-maternal interface. Nat Med. 2006;12(9):1065-74.

22. Vacca P, Cantoni C, Prato C, Fulcheri E, Moretta A, Moretta L, et al. Regulatory role of NKp44, NKp46, DNAM-1 and NKG2D receptors in the interaction between NK cells and trophoblast cells. Evidence for divergent functional profiles of decidual versus peripheral NK cells. Int Immunol. 2008;20(11):1395-405.

23. Rey A, Quartulli F, Escoubet L, Sozzani P, Caput D, Ferrara P, et al. IL-13 induces serine phosphorylation of CPLA2 in mouse peritoneal macrophages leading to arachidonic acid and PGE2 production and blocks the zymosan-induced serine phosphorylation of CPLA2 and eicosanoid production. Biochim Biophys Acta - Mol Cell Biol Lipids. 1999;1440(2-3):183-93.

24. Eyerich K, Dimartino V, Cavani A. IL-17 and IL-22 in immunity: Driving protection and pathology. Eur J Immunol. 2017;47(4):607-14.

25. Hepworth MR, Monticelli LA, Fung TC, Ziegler CGK, Grunberg S, Sinha R, et al. Innate lymphoid cells regulate CD4+ T-cell responses to intestinal commensal bacteria. Nature [Internet]. 2013;498(7452):1137. Available from: http://www.nature.com/doifinder/10.1038/nature12240

26. Maizels RM, Withers DR. MHC-II: A mutual support system for ILCs and T cells? Immunity [Internet]. 2014;41(2):174-6. Available from: http://dx.doi.org/10.1016/j.immuni.2014.07.006

27. von Burg N, Chappaz S, Baerenwaldt A, Horvath E, Bose Dasgupta S, Ashok D, et al. Activated group 3 innate lymphoid cells promote T-cell-mediated immune responses. Proc Natl Acad Sci [Internet]. 2014;111(35):12835-40. Available from: http://www.pnas.org/cgi/doi/10.1073/pnas.1406908111

\section{Figures}


A
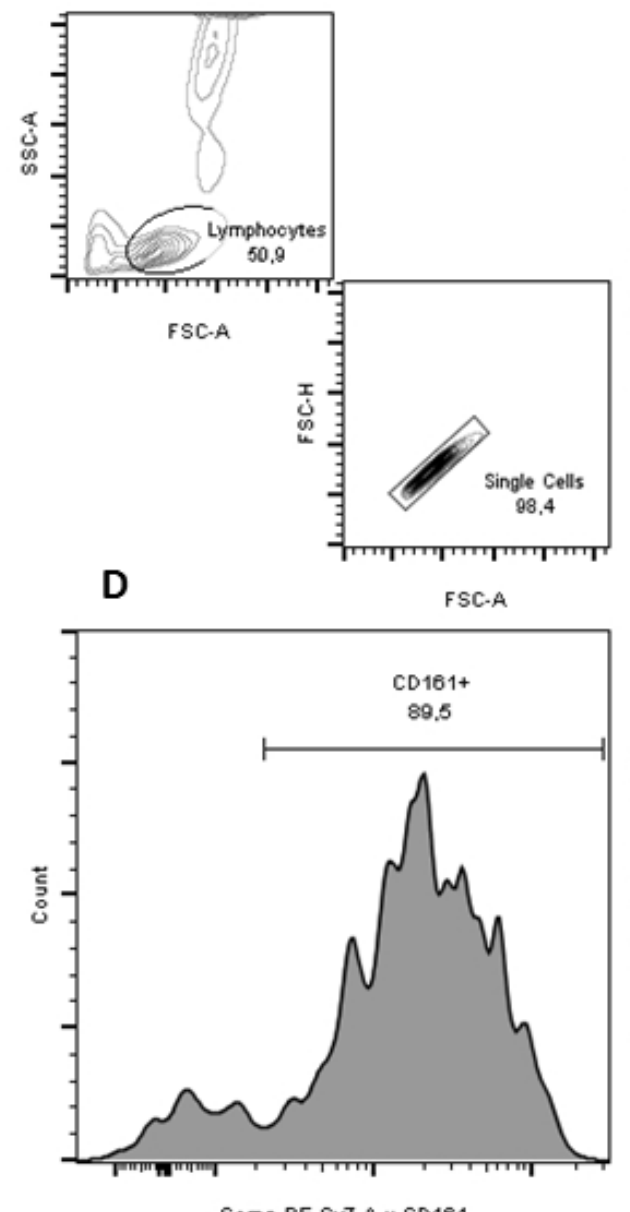

B
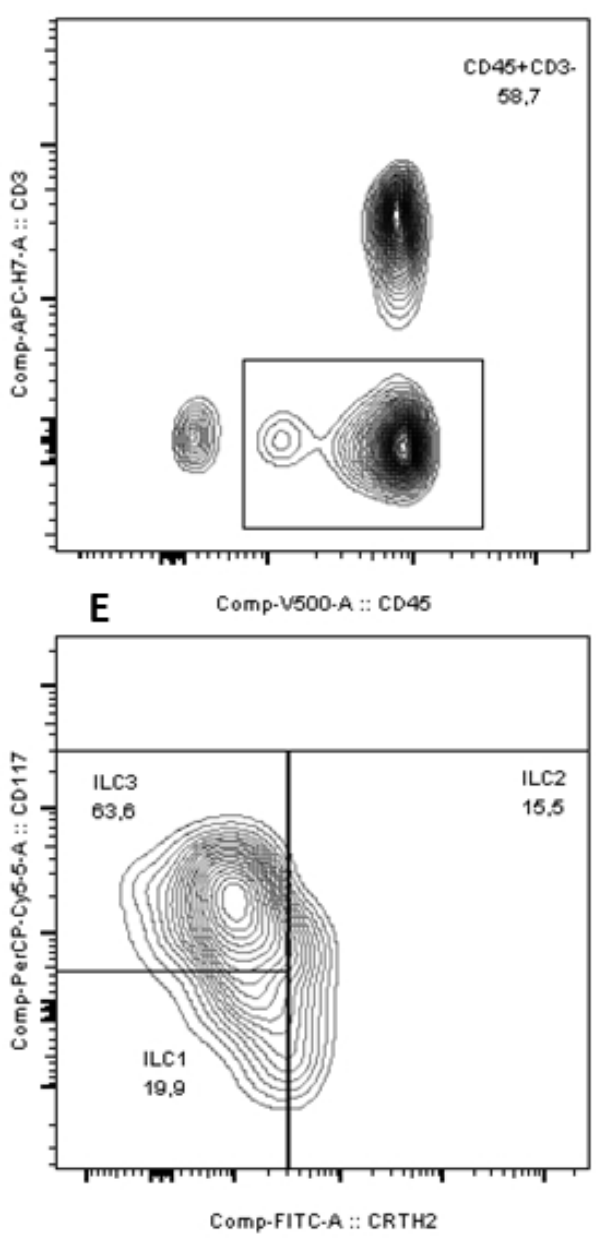

C
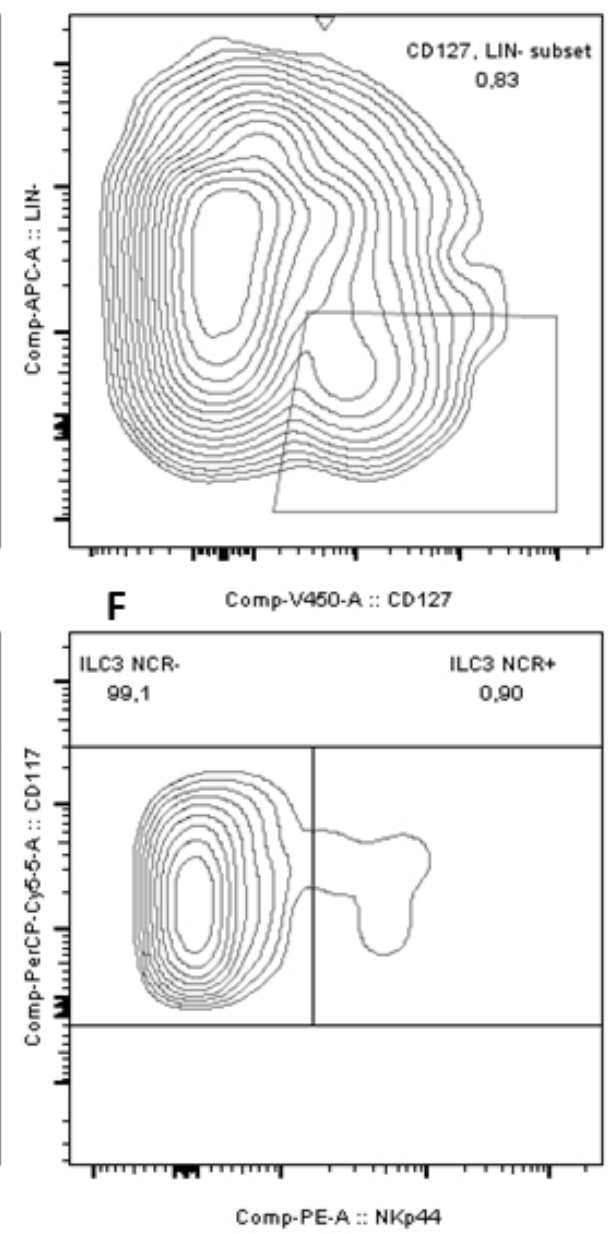

Figure 1

Gating strategy for the identification of ILC3 subpopulations. A. Identification of a lymphocyte population. B. Gating of CD45+ CD3- cells. C. Selection of Lin-CD127+ cells D. Isolation of CD161+ cells. E. Gating ILC3 cells as CRTH2- CD117+. F. Discrimination between ILC3 NCR+ and ILC3 NCR- cells based on NKp44 expression (data analyzed in FlowJo®). 
A
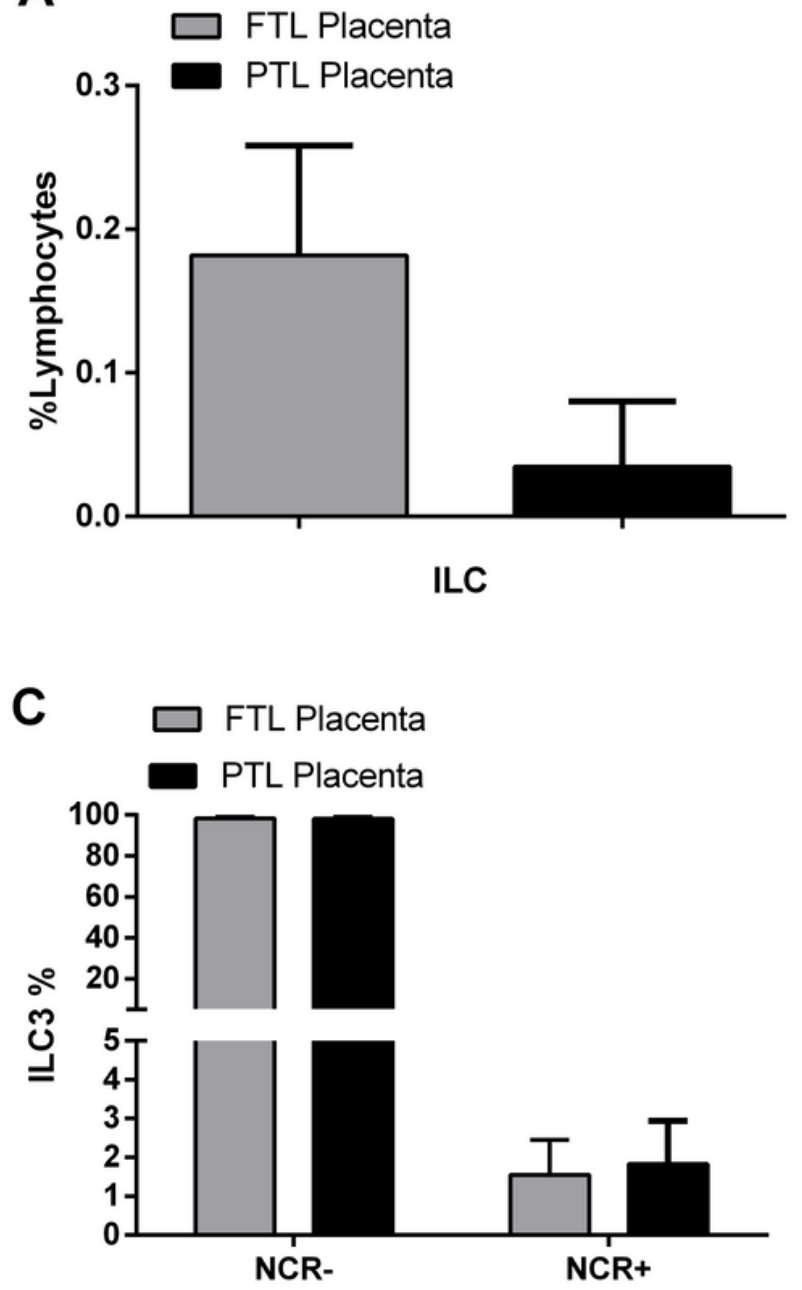

B

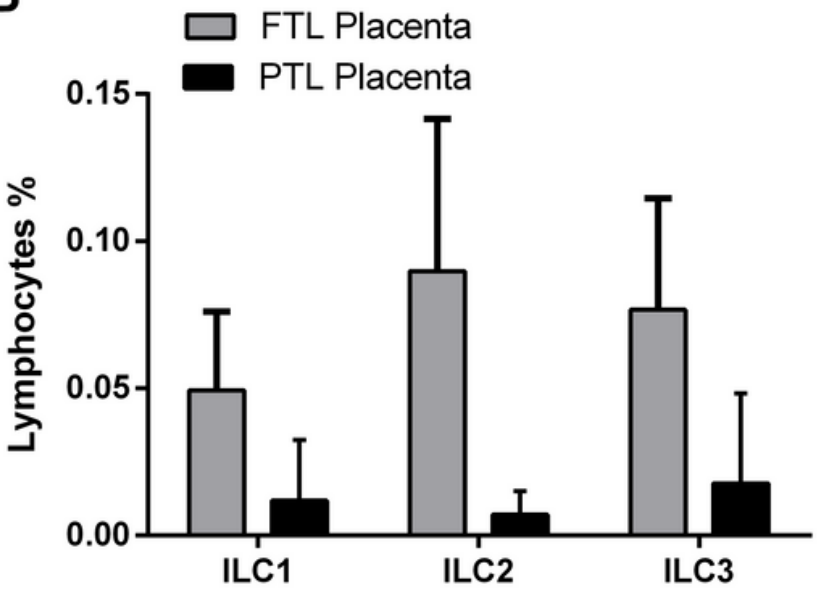

\section{Figure 2}

Graphic displaying the total ILC populations (defined as Lin- CD161+) present within the lymphocyte population in the full-term labor (FTL) samples compared to that in the pre-term labor (PTL) samples $B$. Graphic displaying the relative percentages of the different innate lymphoid cell populations in the FTL and PTL samples within the total lymphocyte population. C. Graphic comparing the two distinct ILC3 populations, NCR+ and NCR- cells, in the FTL and PTL samples. Each data set was analyzed using student t-test analysis with a confidence interval of $95 \%$ using GraphPad® V7 software. 